\title{
Estudio comparativo de los dispositivos vaginales y el extracto de hipófisis equino en la sincronización y superovulación de ovejas
}

\author{
Comparative Study of Vaginal Devices and the Equine Pituitary Extract \\ in the Timing and Sheep Superovulation
}

\author{
F. Rodríguez Gavancho ${ }^{*}$ G. Limaymanta**
}

http://dx.doi.org/10.21503/CienciayDesarrollo.2011.v13.01

\section{RESUMEN}

El propósito del presente estudio fue comprobar la efectividad sincronizadora de dispositivos intravaginales elaborados artesanalmente y comparar la actividad gonadotrófica del extracto de hipófisis equino (EHE) elaborado en el Laboratorio de Reproducción Animal de la Escuela AcadémicoProfesional de Medicina Veterinaria de la Universidad Alas Peruanas de Pachacámac (EAPMV-UAPPachacámac) con la gonadotrofina coriónica equina (eCG) de uso comercial. El trabajo experimental se llevó a cabo en el mes de mayo (época de empadre) del año 2009, en la Cooperativa Agraria de San Francisco de Chichausiri en Junín, ubicada a orillas del Lago Chinchaycocha, aproximadamente a 4000 msnm. Para ello se tomaron 50 ovejas aptas reproductivamente, y se sincronizó el celo con las esponjas vaginales impregnadas con $60 \mathrm{mg}$ de acetato de medroxiprogesterona (MAP). El día en que se colocaron las esponjas vaginales se consideró día cero (0), luego se dividieron las ovejas en dos grupos $\left(\mathrm{T}_{1}\right.$ y $\left.\mathrm{T}_{2}\right)$. Al grupo $\mathrm{T}_{1}$, a partir del día 9 al 12, se le administró vía intramuscular $150 \mathrm{mg}$ de EHE disueltos en $30 \mathrm{ml}$ de agua bidestilada, en doble dosis diaria y en dosis descendentes de 12, 10, 8, 6 y $4 \mathrm{ml}$ por día respectivamente. El día 12 por la mañana se administró además $0,196 \mathrm{mg}$ de tiaprost. Al grupo $\mathrm{T}_{2}$, el día 12 se le administro vía intramuscular 1500 UI de eCG, se retiró la esponja vaginal y se inyectaron $0,196 \mathrm{mg}$ de tiaprost. El día 16 se sacrificaron los animales y se recuperaron los ovarios para verificar la presencia de cuerpos lúteos. Todos los animales presentaron cuerpo lúteo. Las ovejas del $\mathrm{T}_{1}$ presentaron un total de 205 cuerpos lúteos con un promedio de 8,2 $\pm 2,16$; mientras las ovejas del $\mathrm{T}_{2}$ tratadas con eCG presentaron un total de 200 cuerpos lúteos con un promedio de 7,96 $\pm 1,93$. De acuerdo al análisis de varianza (ANVA), no se encontraron diferencias significativas entre la actividad gonadotrófica de ambos tratamientos (EHE y eCG). Se concluyó que las esponjas vaginales elaboradas artesanalmente, cumplen con su función sincronizadora de celo y que el extracto de hipófisis equina (EHE) producido en el Laboratorio de Reproducción Animal de la EAPMV-UAP-Pachacámac tiene actividad gonadotrófica similar a la gonadotropina corionica equina (eCG) comercial.

Palabras clave: gonadotrofina, cuerpos lúteos, hipófisis.

\section{ABSTRACT}

This experiment was conducted in the month of May (breeding season) of 2009, the Agricultural Cooperative of San Francisco de Chichausiri in Junin, located on Lake Chinchaycocha msn approximately $4000 \mathrm{~m}$. The purpose was to test the effectiveness of intravaginal device synchronization and compare

* Docente de la UAP. EAP de Medicina Veterinaria.

${ }^{* *}$ Docente de la UAP. EAP de Medicina Veterinaria. 
handmade gonadotrophic activity, including equine pituitary extract (EHE) and equine chorionic gonadotropin (eCG) for commercial use, for which 50 sheep were taken reproductively fit, zeal were synchronized with vaginal sponges impregnated with $60 \mathrm{mg}$ of progesterone, medroxyprogesterone acetate (MAP). The day on which vaginal swabs were placed was considered day zero (0), then the sheep were divided into two groups or treatments (T1 and T2), treatment (T1), from day 9 to 12 were administered EHE intramuscular $150 \mathrm{mg}$ dissolved in $30 \mathrm{ml}$ of distilled water, in two doses daily dose and descendants (12, 10, 8, 6 and 4) $\mathrm{ml}$ per day, respectively, on 12 days ago also was given $0.196 \mathrm{mg}$ of tiaprost . Treatment two (T2) on day 12 was intramuscularly administered 1500 IU of eCG, vaginal sponge was removed and injected with $0.196 \mathrm{mg}$ of tiaprost. The 16 animals were sacrificed and ovaries were retrieved to verify the presence of corpora lutea. All the animals showed corpus luteum. T1 sheep had a total of 205 corpora lutea with an average of $8.2 \pm 2.16$, while T2 sheep treated with eCG had a total of 200 corpora lutea with an average of $7.96 \pm 1.93$. No significant differences were found between the gonadotrophic activity of both treatments (EHE and eCG). Concluding that the vaginal swabs made by hand, perform their function of synchronizing with zeal and equine pituitary extract (EHE) produced in the Animal Reproduction Laboratory Professional Academic School of Veterinary Medicine, University of Pachacamac Alas Peruanas (EAPMV- UAP-Pachacamac), has activity similar to gonadotrophic equine chorionic gonadotropin (eCG) commercial.

Keywords: gonadotrophin, corpus luteum, pituitary.

\section{INTRODUCCIÓN}

La crianza y la explotación de ovinos localizada en las regiones altoandinas constituyen uno de los reglones más importantes de la producción pecuaria del Perú (1). Se indica que la importancia de la crianza de ovinos en el país es económica, social y ecológica, ya que aproximadamente se tiene 14 millones de ovinos, lo que nos ubica como el segundo país con mayor población ovina en América del Sur, después de Brasil, con aproximadamente una producción de $32 \mathrm{mil}$ toneladas de carne, 12 mil toneladas de lana y 2 millones y medio de pieles por año, generando ingresos económicos para la subsistencia de medio millón de familias campesinas. Además, su crianza está ubicada en las zonas altoandinas, donde se alimenta de pasturas naturales que crecen en 14 millones de hectáreas inapropiadas para la explotación agrícola (2). Por otra parte, el ciclo estrual de la oveja tiene una duración de 17 \pm 2 días, en el que predomina la fase lútea, que se extiende desde el día 2 (el estro es el día cero) hasta aproximadamente el día 13, y una fase folicular que dura desde el día 14 hasta el día 1 del ciclo(3).
Después de la ovulación, el folículo de Graff roto se llena con un coágulo de sangre, lo que se conoce con el nombre de cuerpo amarillo hemorrágico o simplemente cuerpo hemorrágico. Por la influencia de la oleada de la hormona luteinizante $(\mathrm{LH})$, las células de la granulosa de la pared del folículo roto proliferan y se transforman en células luteínicas que subsiguientemente llenan el antro del folículo. A los 4 o 5 días, el cuerpo hemorrágico, que es de color rojo vinoso, se transforma en cuerpo amarillo sólido, llamado también cuerpo lúteo, que es de color rosáceo en el caso de vacunos, aunque algunos pueden ser de color amarillo. Este proceso se conoce con el nombre de luteinización, el cuerpo lúteo o cuerpo amarillo está constituido por dos tipos distintos de células esteroidogénicas, las que contribuyen significativamente a la secreción total de progesterona durante la fase de cuerpo amarillo (fase lútea) del ciclo estrual(4). Respecto de la regulación artificial de la reproducción, esto indica que la estacionalidad del apareamiento limita la tasa de reproducción de la oveja a un parto por año (5). La exposición prolongada de los progestágenos puede producir 
un efecto inhibidor hipotalámico e hipofisiario que disminuye la expresión de la hormona liberadora de gonadotrofinas $(\mathrm{GnRH})$ y la hormona luteinizante $(\mathrm{LH})$ respectivamente, lo cual es un fenómeno utilizado para el control del ciclo estrual en diversas especies.

Tanto la progesterona como los progestágenos, cuando son administrados durante un periodo superior a la duración de un cuerpo lúteo natural, son capaces de inducir un estro bien sincronizado. Sin embargo, la fertilidad del folículo ovulado es menor a la que se obtiene cuando se utilizan durante períodos de 7 a 9 días, debido a que se desarrollan folículos persistentes que ovulan ovocitos envejecidos. Actualmente, la mayoría de los protocolos de sincronización a base de progesterona señalan una duración de 7 a 9 días. La inyección de estradiol (valerato o benzoato de estradiol) al inicio del protocolo tiene como finalidad inducir la regresión del cuerpo lúteo y la atresia del folículo dominante. La inyección de $\mathrm{PGF}_{2} \alpha$ un día antes o al día de retirar la progesterona o el progestágeno induce la luteolisis en aquellos casos en los que este era aún refractario cuando se administró la primera dosis de estradiol.

Robinson, en 1965, fue el primero en comunicareléxitode estasesponjasintravaginales de poliuretano impregnadas con cronolona o fluorogestona (SC-9880) a la dosis de 30-45 mg por 14 a 16 días (6). Los progestágenos más usados son el acetato de fluorogestona (FGA) o cronolona y el acetato de medroxiprogesterona (MAP), con 45 a $60 \mathrm{mg}$, ambos de aplicación intravaginal a través de esponjas impregnadas, y su efectividad es similar (7).

Durante el tiempo que las esponjas impreganadas con progestágeno permanecen en la vagina, se va liberando la hormona que ejerce retroalimentación negativa a nivel del hipotálamo, con la consecuente inhibición de la síntesis y liberación de la hormona liberadora de gonadotropinas ( $\mathrm{Gn} R H)$, y al retirar las esponjas bajan los niveles de la progesterona sérica, reiniciándose la síntesis y liberación de la hormona liberadora de gonadotrofina $(\mathrm{GnRH})$, la cual induce la síntesis y liberación de las hormonas gonadotrofinas: hormona foliculoestimulante (FSH) y hormona luteinizante $(\mathrm{LH})$ por la hipófisis. Las ovejas experimentan el estro en un plazo de 24 a 72 horas. Si al retirar, se administran 350-750 UI de PMSG, se potencializa la sincronización y se adelanta la aparición del estro $(4,5)$.

La superovulación consiste en inducir en forma artificial la producción de un mayor número de ovocitos de los que se liberan en forma natural y característicaparacadaespecie.Estohasidologrado mediante la administración de gonadotrofinas exógenas, las cuales poseen actividades semejantes a la hormona foliculoestimulante (FSH) y a la hormona luteinizante (LH). En el caso de ovinos, esto se logra mediante la administración de gonadotropina coriónica equina (eCG), extracto pituitario anterior equino (EHE) y formas purificadas de la hormona foliculoestimulante (FSH), siendo la eCG una glicoproteína de origen placentario, producida por células de la membrana coriónica del día 40 al 140 de la gestación de la yegua. Esta hormona posee actividad principalmente $\mathrm{FSH}$ y también $\mathrm{LH}$, y tiene unavida media de 40 a 50 horas, además de 118 a 123 horas para sus componentes de rápida y lenta absorción respectivamente(8). Esta gonadotrofina ha sido reportada como una de las hormonas más utilizadas en la inducción de superovulación en ovejas (9). Las dosis superovulatorias son administradas en una sola aplicación, pudiendo variar entre 500 a $2500 \mathrm{UI}(10)$.

Cuando se administra eCG en ovinos, la respuesta media puede ser de 2,8 ovulaciones con la dosis de 700 UI de eCG, y de 9,1 ovulaciones con 1300 UI, administradas en los días 12 o 13 del ciclo estrual (6). 
Las grandes ventajas residen en su bajo costo y su larga vida media (5-6 días). Paradójicamente, su larga permanencia en circulación es la principal desventaja, ya que generan un medio inadecuado para la maduración final de los folículos, además de poder inducir una nueva onda de desarrollo folicular posovulatorio con sus consiguientes niveles elevados de estrógenos, provocando una disminución de la calidad ovocitaria, y por último, al ser un producto purificado a partir de una hormona natural cuya concentración sanguínea varía con el momento de la gestación de la yegua, el efecto biológico del producto comercial varía con la partida utilizada (3).

Otro reporte sobre desventajas en el uso de la eCG menciona una prematura luteinización de folículos, desarrollo de folículos persistentes, reducidos porcentajes de recuperación de ovocitos y fallas de fecundación (11).

Desde 1939, el extracto de hipófisis equina (HAP), conocido también porlassiglas de(EHE), se ha utilizado para inducir superovulación (12). Debido a su alta concentración de FSH, promueve el crecimiento folicular, pero su corta vida media obliga a la administración durante tres días como mínimo. Además, estudios superovulatorios comparativos entre EHE y ECG indican que cuando se mantienen las dosis regulares de EHE se producen pocas variaciones en los rangos de ovulación entre ambos tratamientos; sin embargo, el EHE tiene la ventaja de no inducir prematura luteinización de los folículos, ni formación de folículos persistentes (11).

El objetivo del presente trabajo fue verificar que el extracto de hipófisis equina (EHE) producido en el Laboratorio de Reproducción Animal de la Escuela Académico-Profesional de Medicina Veterinaria de la Universidad Alas Peruanas de Pachacámac (EAPMV-UAPPachacámac) tiene actividad gonadotrófica comparada con la eCG de uso comercial.

\section{MATERIALES Y MÉTODOS}

El presente estudio se realizó en el Laboratorio de Reproducción Animal de la EAPMVUAP y la Cooperativa Agraria de Producción San Francisco de Chichausiri de Junín. Se utilizaron 50 ovejas de la raza Corriedale aptas reproductivamente.

La preparación de las esponjas vaginales impregnadas con progestágenos se realizó de la siguiente forma: a $500 \mathrm{ml}$ de alcohol metílico al $100 \%$ se fueron añadiendo $20 \mathrm{ml}$ de acetona, 5 gotas de cloroformo y $3 \mathrm{~g}$ de acetato de medroxi progesterona; $10 \mathrm{ml}$ de esta solución se agregaron a cada esponja cilíndrica de $3 \mathrm{~cm}$ de diámetro por $5 \mathrm{~cm}$ de largo, en 10 partes de $1 \mathrm{ml}$ por parte, cada 10 minutos, quedando cada una de las 50 esponjas impregnada con $60 \mathrm{mg}$ de progestágeno. Luego se las esterilizó con gas etileno, quedando listas para su utilización.

El método de obtención del extracto hipofisiario equino fue el que se indica (15), es decir, se recuperaron las hipófisis del camal a través de trepanación craneana de equinos sacrificados, se separaron tejidos subyacentes, se pesaron, se trituraron y homogeneizaron en $4 \mathrm{ml}$ de cloruro de sodio $(\mathrm{Na} \mathrm{Cl})$ al $2 \%$ por gramo de hipófisis; luego se centrifugó el homogeneizado por 20 minutos a $1500 \mathrm{rpm}$, se filtró el sobrenadante en lana de vidrio y el residuo se resuspendió en solución de cloruro de sodio para luego ser triturado otra vez. Luego se centrifugó por segunda vez, el sobrenadante se filtró en lana de vidrio. Tras la eliminación del residuo resultante, determinado del volumen total del sobrenadante, se adicionaron 4,2 volúmenes de alcohol etílico de $96^{\circ}$ por 1 volumen de sobrenadante, se lo envasó en un matraz de 1 litro y se lo llevo a reposo por ocho horas a temperatura de refrigeración. Para la recuperación del precipitado formado, primero se eliminó el sobrenadante, luego se filtro el precipitado a través de un embudo Buschner, utilizando como material filtrante una 
lona gruesa colocada en la placa del embudo. El precipitado al ser filtrado fue succionado por una bomba de vacío creado con el efecto Venturi, utilizando un matraz Kitasato, y se lo lavó luego con alcohol de $96^{\circ}$ y posteriormente con éter. Terminado el filtrado, fue secado al vacío en una campana de vidrio con silica gel. Posteriormente, el precipitado seco se pulverizó en mortero y se lo almacenó en refrigeración. Para su utilización se lo suspendió en agua destilada estéril.

De 150 hipófisis colectadas del camal, se obtuvieron 4,4 g de HAP. Esto significa que para 1 gramo de HAP se han requerido 33 hipófisis, $y$ como se utilizaron $150 \mathrm{mg}$ de EHE por animal, se necesitaron aproximadamente 5 hipófisis para superovular 1 oveja. Las hipófisis colectadas alcanzaron solo un peso promedio de $0,8 \mathrm{~g}$, con un rango de 0,5 a $1,6 \mathrm{~g}$.

Para la sincronización del celo y la superovulación, a cada oveja se le colocó dentro de la vagina una esponja impregnada con progestágeno, indicando el día de colocación de esponja como día cero (0). Luego se administró $1 \mathrm{mg}$ de benzoato de estradiol por vía intramuscular y se dividieron las ovejas en dos grupos o tratamientos $\left(\mathrm{T}_{1} \mathrm{y}_{2}\right)$.

A los animales del grupo 1 o $\mathrm{T}_{1}$, a partir del día 9, se les administraron por vía intramuscular 150 mg de EHE resuspendidos en $30 \mathrm{ml}$ de agua por animal, durante 4 días (día 9, 10, 11 y 12), a doble dosis diaria (mañana y tarde) y en dosis descendentes (12 ml, $8 \mathrm{ml}, 6 \mathrm{ml}$ y $4 \mathrm{ml}$ ). En la mañana del día 12 se retiró la esponja vaginal y se inyectaron $0,196 \mathrm{mg}$ de tiaprost (análogo de PGF2 $\alpha$ ), y por la tarde se aplicó la ultima dosis de EHE.

A los animales del grupo $2\left(\mathrm{~T}_{2}\right)$, el día 12 por la mañana después del retiro de la esponja vaginal, se les administraron 1500 UI de gonadotrofina coriónica equina (eCG) de uso comercial e inyectaron $0,196 \mathrm{mg}$ de tiaprost.

\section{RESULTADOS Y DISCUSIÓN}

Para evaluar la respuesta gonadotrófica en ambos tratamientos aplicados, se procedió a sacrificar a todos los animales el día 16. Luego, se recuperaron los ovarios para verificar la presencia de cuerpos lúteos hemorrágicos fácilmente reconocibles por el color rojo vinoso que presentan, tanto en el ovario izquierdo como en el derecho de cada animal, como respuesta superovulatoria a las gonadotrofinas administradas.

Tabla 1. Número de cuerpos lúteos observados por tratamiento.

\begin{tabular}{|c|c|c|}
\multirow{2}{*}{ Tratamiento } & \multicolumn{2}{|c|}{ CUERPOS LÚTEOS } \\
\cline { 2 - 3 } & Total & Promedio \\
\hline Tratamiento con EHE & 205 & 8,2 \\
\hline Tratamiento con eCG & 200 & 7,96 \\
\hline
\end{tabular}

De la observación del cuadro 1 , se concluye que los animales del $\mathrm{T}_{1}$ presentaron un total de 205 cuerpos lúteos con un promedio de $8,2 \pm 2,16$ cuerpos lúteos, como respuesta a la actividad gonadotrófica de la EHE, mientras las ovejas del $\mathrm{T}_{2}$, tratadas con eCG, presentaron un total de 200 cuerpos lúteos, con un promedio de 7,96 $\pm 1,93$ cuerpos lúteos. Resulta evidente también que no se encontraron diferencias estadísticas significativas entre la actividad gonadotrófica de ambos tratamientos (EHE y eCG), puesto que el $F_{c} \leq F_{t}$. Debemos, asimismo, destacar que estos resultados son parecidos a los reportados por Gatica R. (12), que reporta haber obtenido 139 cuerpo lúteos de 15 ovejas tratadas con EHE de caballo y logrado un promedio de 9,27 cuerpos lúteos por oveja y un rango de 3 y 14, en tanto que para el caso de eCG reporta 116 cuerpos lúteos de 15 ovejas, con un promedio de 7,73 cuerpos lúteos y un rango de 2 y 13 . Estos resultados son semejantes a los reportados por $(3,6,11)$. 


\section{CONCLUSIONES}

En conclusión, se demuestra que la sincronización de celo mediante las esponjas vaginales elaboradas artesanalmente funcionó bien, ya que no hubo una sola oveja que no respondiera a las gonadotrofinas administradas. Además, se demostró que el extracto de hipófisis equina (EHE) producido en el Laboratorio de Reproducción Animal de la Escuela de Académico-Profesional de Medicina Veterinaria de la Universidad Alas Peruanas de Pachacámac (EAPMV-UAP-Pachacámac) tiene actividad gonadotrófica semejante a la gonadotropina coriónica equina (eCG) comercial.

\section{REFERENCIAS BIBLIOGRÁFICAS}

1. Montesinos M.E., Alencastre R., Quijandría B., Blackwell R. Selección de carneros Corriedale mediante la aplicación de pruebas de performance y progenie en el Centro Experimental Chuquibambilla. Univ. Nac. del Altiplano,Puno, Perú, 1991.

2. Aliaga G.J.,Producción deovinos. Universidad Nacional Agraria, Facultad de Zootecnia, Ed. Juan Gutenberg, Lima, Perú, 2006.

3. Rubianes E., Castro T., Vigñoles C., Ungerfeld R., Carbajal B., Kmaid S. Superovulación y transferencia embrionaria en ovinos. Departamento de Fisiología, Facultad de Veterinaria, Montevideo, Uruguay, 1995.

4. Hafez E.S.E., Hafez B. Reproducción e inseminación artificial en animales. $7^{\mathrm{a}} \mathrm{ed}$. Mexico, Ed. Mc Graw Hill Interamericana, 2002.

5. Burgos R., HanckeJ. “Estrógenos, andrógenos y progestágenos”. En: Botana L., Landoni F., Jimenez T. Farmacología y terapeutica veterinaria. $2^{a}$. ed., México, Ed. McGrawHill Interamericana, 2002, p. 411-434.

6. Hunter R.H.F. Fisiología y tecnología de la reproducción en la hembra de los animales domésticos. España, Ed. Acribia,1980.

7. Intervet. Compendium de reproducción animal. $9^{a}$ ed., Ed. Sinervia, UruguayParaguay, 2007.

8. Letelier C. Efectos del origen de la FSH en la inducción de superovulación en ovejas. (Tesis de licenciatura en medicina veterinaria). Valdivia, Chile, Universidad Austral de Chile,1997.

9. Evans G., Robinson T. "The Control of Fertility in Sheep: Endocrine and Ovarian Responses to Progestagen-PMSG Treatment in the Breeding Season and in Anoestrus".J Agric Sci (Cambridge), 1980; 94: 69-88.

10. Whyman D., Moore R. "Effects of PMSG and the Prostaglandin F2 Alfa Analogue, Cloprostenol, on Superovulation, Fertilization and Egg Transport in the Ewe". JReprod Fertil, 1980; 60: 267-72.

11. Moore N.W., Shelton J.N. "Response of the Ewe to a Horse Anterior Pituitary Extract".J Reprod Fertil, 1964; 7: 79-87.

12. Gatica R. Hormonoterapia reproductiva. VII Jornadas Latinoamericanas de Buiatría. Valdivia, Chile, 1985.

13. Martínez J. W. Evaluación del extracto de bipófisis equino (EHE) en la superovulación deovejas. (Tesis para optar el título de médico veterinario). Lima, Perú, Universidad Alas Peruanas, 2003.

14. Zuzuki M. Actividad gonadotrópica del extracto hipofisiario equino (EHEc). (Tesis para optar el título de médico veterinario). Lima, Perú, Universidad Alas Peruanas, 2003.

15. Araya J. C., Gatica R., Del Campo M. R. Inducción de superovulación en hembras bovinas utilizando un extracto hipofisiario equino (EHE). VII Jornadas Latinoamericanas de Buiatría, Valdivia, Chile, 1985. 